\title{
Understanding And Restoring Dopaminergic Function In Fibromyalgia Patients Using A Mindfulness-Based Psychological Intervention: A [18F]-DOPA PET Study Study Protocol For The FIBRODOPA Study- A Randomized Controlled Trial
}

Katharina Ledermann ( $\sim$ katharina.ledermann@usz.ch )

UniversitatsSpital Zurich https://orcid.org/0000-0001-9355-2979

Roland von Känel

UniversitatsSpital Zurich

Chantal Berna-Renella

Centre Hospitalier Regional Universitaire de Lille Pole de Biologie Pathologie Genetique

Haiko Sprott

University of Zurich, Schmerzpraxis Hottingen

Josef Jenewein

Universitatsklinikum Schleswig Holstein Campus Kiel Klinik fur Innere Medizin II mit den Schwerpunkten Hamatologie und Onkologie

Eric Garland

University of Utah College of Social Work

Chantal Martin-Sölch

Universite de Fribourg Faculte des lettres

Study protocol

Keywords: Fibromyalgia, Mindfulness-Oriented Recovery Enhancement, clinical pain, Positron Emission Tomography

Posted Date: June 28th, 2021

DOl: https://doi.org/10.21203/rs.3.rs-47401/v1

License: (c) (i) This work is licensed under a Creative Commons Attribution 4.0 International License. Read Full License 
Version of Record: A version of this preprint was published at Trials on December 1st, 2021. See the published version at https://doi.org/10.1186/s13063-021-05798-1. 


\section{Abstract}

Background: (FM). Fibromyalgia (FM) is a very prevalent and debilitating chronic pain disorder that is difficult to treat. Mindfulness-based techniques are regarded as a very promising approach for the treatment of chronic pain and in particular FM. The Mindfulness-Oriented Recovery Enhancement (MORE) intervention, a mindfulness-based group intervention, has shown beneficial effects in opioid-treated chronic pain patients, including reduced pain severity, functional interference, and opioid dosing, by restoring neurophysiological and behavioral responses to reward. First evidence for a hypodopaminergic state and impaired reward processing in FM have been reported. However, little is known about its impact on dopamine (DA) function and in particular with regard to DA responses to monetary reward in FM. The aim of the present study protocol is to evaluate if MORE is able to restore the DA function in FM patients, in particular with regard to the DA responses to reward, and to reduce pain and mood complaints in FM.

Methods/design: The present study is a multi-center RCT with 3 time points: before the intervention, after completion of the intervention and 3-months after completion of the intervention. Eighty FM patients will be randomly assigned to either the MORE intervention $(\mathrm{N}=40)$ or to a wait-list control group $(\mathrm{N}=40)$. Additionally a comparison group of healthy women $(\mathrm{N}=20)$ will be enrolled. The MORE intervention consists of eight 2-hour long group sessions administered weekly over a period of 8 weeks. Before and after the intervention, FM participants will undergo [18F] DOPA Positron Emission Tomography (PET) and functional MR imaging while performing a monetary reward task. The primary outcome will be endogeneous DA changes measured with [18F] DOPA PET at baseline, after the intervention (after 8 weeks for control group), and at 3 months' follow-up. Secondary outcomes will be (1) clinical pain measures and FM symptoms using standardized clinical scales 2) functional brain changes 3) measures of negative and positive affect, stress and reward experience in daily life using the Experience Sampling method (ESM) 4) biological measures of stress including cortisol and alpha-amylase.

Design: If the findings of this study confirm the effectiveness of MORE in restoring DA function, reducing pain and improving mood symptoms, MORE can be judged to be a promising means to improve quality of life in FM patients. The findings of this trial may inform health care providers about the potential use of the MORE intervention as a possible non-pharmacological intervention for FM.

Trial registration: ClinicalTrials.gov NCT 04451564. Registered on 3 July 2020. The trial was prospectively registered.

\section{Introduction}

\section{Background and rationale}

Fibromyalgia (FM) is a chronic, painful, musculoskeletal disorder characterized by widespread pain, accompanied by a broad spectrum of associated somatic and psychological manifestations, including fatigue, sleep disturbances, stiffness, anxiety and cognitive dysfunction (Wolfe et al., 2011). It is one of the most prevalent chronic pain conditions (Thieme, Mathys, and Turk, 2017) with an estimated 
prevalence between $0.5 \%$ and $4 \%$ with a ratio of $3.5 \%$ in women to $0.5 \%$ in men (Jones et al., 2015). Like other chronic pain conditions, FM often leads to disability, affective disturbance and poor quality of life and is also associated with high direct and indirect disease related costs (Penrod et al. 2004). The etiology of FM is largely unknown. However, several factors appear to underlie the disorder, including dysfunction of the central nervous system (CNS) and autonomic nervous systems, neurotransmitters, hormones, immune system, as well as external stressors and psychological factors (Bellato, 2012). More generally, chronic pain is commonly associated with comorbid affective disorders (e.g. anxiety, depression) and cognitive deficits (e.g. memory impairment), suggesting on one hand critical involvement of higher order neural brain processing (Bushnell, Ceko \& Low, 2012), and on the other the necessity to develop specific interventions targeting comorbid mental disorders, mood and cognitive dysfunctions (see for instance (de Jong et al. 2017). Among the neural changes observed in chronic pain, there is increasing evidence for alterations in the dopaminergic (DA) system. Evidence supporting a hypodopaminergic state in chronic pain comes from both preclinical (Niikura, Narita, Butelman, Kreek, \& Suzuki, 2010) and clinical data (Hipolito et al., 2015; Loggia et al., 2014). Furthermore, accumulating evidence suggests that the mesolimbic DA system modulates the perception of nociceptive information, and the affective symptoms of chronic pain (Baliki, Geha, Fields, \& Apkarian, 2010). Taken together, there are now multiple lines of evidence showing that chronic pain, including FM, leads to a hypodopaminergic state that results in enhanced pain sensitivity and might impair positively motivated behavior (Taylor, Becker, Schweinhardt, \& Cahill, 2016). In addition, DA is involved in descending inhibitory modulation of pain transmission, which is an additional link between hypodopaminergia and chronic pain (Potvin, Grignon, \& Marchand, 2009). In a previous project of our group that aimed at investigating DA function in FM, we found a reduced DA function in FM patients, and showed group differences in DA receptor binding in striatal regions between FM participants with and those without depression compared to healthy subjects (Ledermann et al., 2016). In addition, we found a differential modulation of pain by DA in healthy controls and FM participants (Ledermann et al., 2016). Findings from functional neuroimaging studies indicate that a network of brain regions, including the orbitofrontal cortex, the ventral (specifically the nucleus accumbens, Nacc) and dorsal striatum, the amygdala and the anterior cingulate gyrus forming the so-called reward system(O'Doherty, 2004). In chronic pain, alterations in brain structural features, functional connectivity, or activity of these regions have been reported, compared to healthy controls, or people who tend to recover from acute pain (Baliki et al., 2010; Hashmi et al.,2013; Wood, Patterson et al, 2007). The neural changes observed in regions associated with the brain reward system could provide a possible explanation for the high incidence of comorbid affective disorders in chronic pain patients (Ledermann et al., 2017). In conclusion, the exact mechanisms by which the brain reward networkmodulates chronic pain havenot been established yet. More specifically, although there is first evidence for a hypodopaminergic state in FM (Ledermann et al., 2016; Wood, Patterson, et al.,2007; Wood, Schweinhardt et al., 2007), the effects of chronic pain on the ability to enjoy rewards and hence anhedonia have insufficiently not been investigated. Finally, the exact role of DA in the modulation of chronic pain remains unclear. On another note, the current evidence-based guidelines for the treatment of patients with FM are inconsistent (Thieme et al., 2016). Recent meta-analyses conclude that optimal treatment interventions should include components aimed at enhancing adaptive cognitive and 
behavioural responses (Aman, 2018; Goldenberg, Burckhardt, \& Crofford, 2004), and broad improvements have been observed with treatment plans that include non-pharmacologic interventions (Aman, 2018). This is in line with the current international guidelines that recommend aerobic exercise, cognitivebehavioural therapy (CBT), and multicomponent treatment as first choice for the care of FM patients (Thieme et al., 2016).

A growing body of research has demonstrated that mindfulness-based interventions are clinically effective for a wide range of conditions (for a review see Goyal et al. (Goyal, Singh, Sibinga, 2014) leading to them increasingly being used for the treatment of chronic pain conditions including FM (Rosenzweig et al., 2010; Veehof, Trompetter, Bohlmeijer, \& Schreurs, 2016). A recent systematic review indicates that mindfulness-based interventions produce statistically significant, moderate effect size improvements in mood-related outcomes in FM (Theadom et al., 2015). However, most studies of mindfulness-based interventions have concentrated on negative affective-related constructs, and have not given sufficient attention to the effects of mindfulness on positive affect and reward (Garland et al. 2015).

In contrast, Mindfulness-Oriented Recovery Enhancement (MORE) is a mind-body intervention specifically designed to enhance positive emotion regulation and natural reward processing by uniting complementary aspects of mindfulness training, CBT and positive psychological principles into an integrative treatment strategy. MORE was originally designed as a behavioral medical intervention for addictive behaviors (Garland et al., 2010; Garland, 2013; Garland et al., 2016), but was more recently adapted to address chronic pain among individuals receiving long-term opioid analgesic therapy. Two Stage 2 randomized clinical trials (RCTs, $N=115$ and $N=95$ ) showed that MORE significantly reduces pain severity and pain-related functional interference (Garland, Manusov et al., 2014, 2019), as well as opioid dosing (Garland et al., 2020), among chronic pain patients. In addition, the effects of MORE on reducing pain severity and opioid misuse were associated with increases in positive emotional processes, including positive affect, savoring, and meaning in life (Garland et al., 2017; Garland et al., 2019). In addition to these psychological effects, randomized controlled studies demonstrate that MORE is also associated with behavioral and neurophysiological changes in reward processing (Garland, Froeliger, et al., 2014; Garland et al., 2015), including increases in the late positive potential of the EEG and enhanced corticostriatal activity during savoring of natural rewards (Garland et al., 2019; Froeliger et al., 2017), suggesting that MORE might improve clinical outcomes by enhancing reward system function. It is however not clear whether or not the experimental modification of reward experiences represents a mechanism of change in MORE. Because our previous results showed dysfunctional DA responses to rewards in FM (Ledermann et al., 2017), the use of MORE in this group of patients could be very promising. Furthermore, no study so far has investigated the molecular underpinnings of MORE on the dopaminergic reward system.

In addition, daily measures of self-reported stress experiences and self-reported pain symptoms in FM indicate that stress exacerbates the pain feelings in everyday life of FM patients (Fischer et al., 2016). Further, it has been hypothesized that FM patients may find it more difficult to mount a resilient affective response to stressful events if the force of negative affect compromises their resources of positive affect 
(Zautra, Fasman, et al., 2005). In turn, positive affect (PA) is a source of resilience against pain and negative affectivity (Zautra, Johnson, \& Davis, 2005); and momentary positive emotions, rather than more general satisfaction with life, are associated with increased psychological resilience (Cohn, Fredrickson, Brown, Mikels, \& Conway, 2009), for instance against later affective symptoms in participants vulnerable to depression (Geschwind et al., 2010). In addition, in FM, deficits in positive affect regulation have been reported (Zautra, Fasman, et al., 2005). Using ecological momentary assessments, MORE and mindfulness have been shown to significantly boost positive affect regulation, momentary positive emotions, and enhance responsiveness to pleasant daily life activities (Garland et al. 2017; Garland et al., 2019; Geschwind et al., 2010).

Taken together, as MORE seems very promising in being able to restore DA functionality of the DA system in FM patients and enhancing natural reward processing, here we propose a RCT to examine whether MORE can restore the functionality of the DA reward system in FM patients by comparing the neural responses to monetary reward in a group of FM patients and healthy controls with 18F-DOPA PET and fMRI before and after the 8-week MORE intervention. The effect of MORE will be tested against a wait-list control condition. Secondary outcomes are clinical effects of the MORE intervention on pain and other FM related symptoms. Further, we will test whether the MORE intervention impacts self-reported measures of momentary pain, mood, stress and reward experience in everyday life measured by the Experience Sampling method (ESM) as well as biophysiological measures of stress, including cortisol and alpha-amylase.

\section{Methods / Design}

\section{Aims and objectives}

The aim of the present study protocol is to evaluate if MORE is able to restore DA function in FM patients, in particular with regard to the DA responses to reward, and to reduce pain and negative mood in FM.

The objectives are as follows:

1. To compare the 18F-DOPA influx in striatal regions and neural responses to reward with fMRI before the MORE intervention between FM patients and a group of healthy controls

2. To compare the 18F-DOPA influx and neural responses to reward measured with fMRI after the MORE intervention between FM patients that participated in MORE versus those assigned to a waitlist control condition.

3. Explore changes in FM related pain and mood symptoms after the MORE intervention

4. Explore changes in self-report measures of behavioral and biological stress in FM patients after the MORE intervention

Our hypothesis are: 
1. FM patients show a lower $18 \mathrm{~F}-\mathrm{DOPA}$ influx in striatal regions and decreased neural response to reward measured with $\mathrm{fMRI}$ than a healthy control group before the MORE intervention

2. FM patients participating in the MORE intervention show an increased 18F-DOPA influx and increased neural responses to reward measures with $\mathrm{fMRI}$ after the MORE intervention compared to a wait-list control group.

3. We expect FM patients participating in the MORE intervention to show significant changes in pain, mood and FM related outcomes compared to a wait-list control group.

4. We expect changes in everyday self-report measures of stress and pain levels including biological stress measures in FM patients versus healthy controls before the MORE intervention.

\section{Trial Design}

This is a multi-center RCT with 3 time points: before the intervention (pre-test measures, T1), after completion of the intervention (post-test measures, T2) and 3-months after completion of the intervention (follow-up measures, T3) (see Fig. 1). Participants in the wait-list control condition will perform the T1 and T2 measures adjuvant eight weeks apart. Study evaluation will be done by comparing within and between the groups. Healthy controls will perform T1 measures only. The potential effect will be assessed on a series of outcome measures (Table 1). Measures will take place at baseline (T0), T1 T2 and T3. Before T1, we will perform the screening of the participants with regard to the inclusion and exclusion criteria (T0). Pre-test and post-test measures include each a 18F-DOPA PET scan at rest and a fMRI measure with use of the reward task, the ambulatory assessment (AA) measures, the clinical and pain measures as well as the fMRI measures. The follow-up consists in questionnaires assessments only, including the self-reported pain and clinical measures associated with PET and fMRI meausres. The questionnaires will be presented through an online survey that is suitable for RCT's (Research Electronic Data Capture, redcap) and available at the Universities Fribourg,and that can be filled at home. The measures that are not self-reported will be performed by trained assessors, who will be blind to the treatment condition to minimize expectation bias of the assessors.

The wait-list control will undergo the same measures at pre-test and post-test, with a post-test planned on average after 8 weeks. They will not participate in any specific intervention, but will be given the opportunity to participate in MORE after the follow-up assessment.

The study is a multi-center study and includes the University Fribourg, Department of Psychology; the University Hospital Lausanne (CHUV), Center for integrative and complementary medicine \& Pain Center, and University Hospital Zurich, Department of Consultation-Liaison Psychiatry and Psychosomatic Medicine. The intervention will be given in each of these centers in order to access a larger pool of participants. The PET-scans will be performed at the PET-Center of the Department of Nuclear Medicine at the University Hospital Zurich. The fMRI measures will be performed at the Department for Neuroradiology at the University Hospital Zurich.

\section{Eligibility criteria}


The following groups of participants will be included: a) 80 female subjects fulfilling the classification criteria of the American College of Rheumatology for FM (ACR 2011 criteria) (Wolfe, 2010) and without any psychiatric condition according to the ICD-10 and b) 20 healthy women without a history of chronic pain or any mental disorder for the PET-measures; and a total of 15 healthy women for the daily assessments. The groups will be matched on age. All participants will be right-handed and older than 18 years old. Subjects will be excluded if they are pregnant, if they have a history of neurological disorders, current substance or tobacco abuse, current and past substance dependence, schizophrenia spectrum disorder, any other form of chronic pain (except FM for the FM group), and if they have been treated with medication affecting the central DA system in the three months preceding the scanning session (including opioids, neuroleptics, antileptics, antidepressants and lithium). Participating in another RCT or some form of individual or group psychotherapy focusing on pain management is prohibited.

\section{Recruitment process}

Patients will be recruited from the interdisciplinary outpatient clinic for pain at the University Hospital Zurich, from the Department of Consultation-Liaison Psychiatry and Psychosomatic Medicine at the University Hospital Zurich, from the Zugerklinik, from the Neurology Department and interdisciplinary outpatient clinic for pain at the Canton Hospital Fribourg, from the outpatient pain clinic at the Divisiont of Anesthesiology as well as the service of rheumatology at the Lausanne University Hospital, from private practices, and from adds on websites of the Rheumaliga, as well as patient groups such as the Fibromyalgieforum Schweiz in the German-speaking and French-speaking parts of Switzerland. The healthy controls will be recruited through ads, word of mouth and from our previous and current studies as well. When interested participants call into one of the study telephone lines, they will be told about the study and screened for eligibility using pre-determined scripts. If potentially eligible, an in-person visit (90 min duration) at the study center will be scheduled. This first visit can be the start of the study, or this start can be reported, depending on the clinical situation. At study start, eligibility will be confirmed, consent provided and the initial assessment completed.

\section{Randomization}

The 80 FM participants will be assigned to the MORE treatment or to a wait-list condition in a randomized way (see flow-chart, Fig. 1). The randomization sequence is generated by the website Research Randomizer (http:// www.randomizer.org). The list is concealed by instructed office personnel in the study center of the University Fribourg, Switzerland, who is unaware of any patient information. The randomized participants will join the MORE group or wait-list control. Participants will not be blinded regarding assignment to intervention. Group numbers should not exceed a maximum of 12 per group. As the study progresses, participants coming off the waitlists will also be added to the ongoing groups. We will begin with one MORE group running in Zurich and in Fribourg, but will add additional groups as numbers require.

\section{Description of the MORE Intervention}


On the basis of the MORE treatment manual (Garland, 2013), sessions will offer instruction in applying mindfulness and related skills to the following topics: discriminating between nociception, pain, and suffering; gaining awareness of automaticity and coping habits in chronic pain; disrupting the link between negative emotions, catastrophizing, and pain experience through reappraisal; refocusing attention from pain and life stressors to savour pleasant experiences; cultivating self-transcendence and meaning in life; and developing a mindful recovery plan. For this Trial the original MORE session 5 targeting opioid craving will be modified to address mindful self-care strategies. Mindfulness training will involve mindful breathing and body scan techniques, with an emphasis on developing metacognitive awareness and shifting attention from affective to sensory processing of pain. During this process, patients are taught to decompose pain experience into its constituent sensations (e.g., heat, tightness, tingling), as well as to increase awareness of the center, edges, and permeability (versus solidity) of these sensations, and then to notice and savor any adjacent or distal pleasant sensations (Garland, 2020). Sessions will be video-taped for control of therapists' adherence with the manual. MORE participants will be asked to engage in daily 15-minute mindfulness, reappraisal, and savoring practice sessions at home guided by a MP3 file.

\section{Measures}

A detailed overview of questionnaires and measures is given in Table 1. Schedule of enrolment, interventions, and assessments is provided in Table 2. Data management including data entry, coding, security and storage will be provided by the web-based application Research Electronic Data Capture (REDCap) http://dx.doi.org/10.5195/jmla.2018.327 that is available at the University of Fribourg. It is Health Insurance Portability and Accountability Act (HIPAA)-compliant and highly secure.

\section{Socio-demographic data}

Data to be collected at baseline include age, sex, marital status, education level, professional status.

Measures of functioning includes a measure of quality of life with the World Health Organization quality of life questionnaire WHOQOL Brief questionnaire (Skevington, 2004) and the Fibromyalgia Impact Questionnaire-Revised (FIQ-R; Bennett, 2009) that also includes measure of fatigue. Measures of sleep quality include the medical outcomes study sleep scale (MOS; (Stewart, 1988 Hays, 1992) that has been widely used in rheumatology research and is recommended by OMERACT-10.

\section{Questionnaires}

\section{Pain related outcomes:}

- Pain severity and functional interference The Brief pain inventory (BPI) is used to obtain information on self-reported measures of pain severity and functional interference using the Brief pain inventory (BPI, Cleeland, 1994). Pain magnitude is queried by four items that ask about pain now, worst pain, least pain and average pain. Items use numeric rating scales anchored by 0 (no pain) to 10 (most severe pain). Pain interference consists of seven items that ask about how pain interferes with 
aspects of daily living using numeric rating scales anchored by 0 (no interference) to 10 (completely interferes).

- Current pain intensity The one item Verbal Rating Scale (VRS) from the SF-36 (Ware, 1992) is used to measure current pain intensity. The VRS has proven itself over decades as a valid, reliable and change sensitive measure of subjective pain (Ware, 1992).

- Pain interference with functional impairment To measure the degree to which pain interferes with function in major life areas, we will use the 7-item Pain Disability Index (PDI) (Dillmann, U. et al.,1994).

\section{Other clinical measures:}

- Severity of depressive symptoms To assess the severity of depressive symptoms, the Beck Depression Inventory (BDI)-II (Hautzinger, 1991, Beck, 1961), a self-report questionnaire will be used.

- State and Trait Anxiety The State-Trait Anxiety Inventory (STAI) will be used as a measure of state and trait anxiety (Laux, 1981).

- Mood states The Profile of Mood States (POMS) (McNair et al., 1971) will be used to assess transient, distinct mood states. The POMS measures six different dimensions of mood swings over a period of time with high sensitivity to change.

- Quality of Life To measure quality of life, we will use the quality of live scale from the World Health Organization WHOQOL Brief questionnaire consisting of 26-items, (Skevington et al. 2004). The WHOQOL Brief has good to excellent psychometric properties of reliability and performs well in preliminary tests of validity (WHOQOL group, 1998).

- Function, Impact and Overall symptoms of Fibromyalgia The revised Fibromyalgia Impact Questionnaire (FIQ-R) (Bennet, 2009) is a commonly used and validated 9-item instrument in the evaluation of fibromyalgia (FM) patients.

- Sleep quality will be assessed using the Sleep quality, medical outcomes study sleep scale MOS (Stewart, 1988, Hays 1992) that includes 12 items assessing sleep disturbance, sleep adequacy, somnolence, quantity of sleep, snoring, and awakening short of breath or with a headache.

- Mechanisms underlying the MORE intervention Mechanisms related to the MORE intervention we will assess with the following questionnaires: Nonreactivity: Five Facet Mindfulness Questionnaire (Baer et al., 2006) the reinterpretation of pain sensations (Subscale of Coping Strategies Questionnaire, (Brown \& Nicassio, 1987), positive reappraisal (Subscale of Cognitive Emotion Regulation Questionnaire CERQ) (Garnefski et al., 2007), and savouring (Savouring Beliefs Inventory) (Bryant et al., 2003), according to (Garland et al., 2014). They aim to understand the mechanisms related to the MORE intervention.

\section{PET / fMRI measures}

Non-invasive 18 F-DOPA PET and fMRI imaging of the brain (high resolution, anatomical, T1 weighted imaging, resting state will be used to study presynaptic and functional changes in the brain. 
PET images will be acquired at the Department of Nuclear Medicine at the University Hospital Zurich. MRI overlay images will be acquired at 3 Tesla at the Department of Neuroradiology at the University Hospital Zurich, using T1 weighted sequence (MP-RAGE) to provide an anatomical framework for image analysis. 18-F DOPA is a well-validated measure of presynaptic DA function (Heiss and Hilker, 2004); and previous studies showed differences in 18-F DOPA binding between FM and healthy participants at rest (Wood, 2007). PET-measures will be performed before and after the MORE intervention (after 8 weeks for the wait-list control group). One hour before scanning they will receive as routinely an oral dose of Carbidopa 100mg, a peripheral DOPA decarboxylase blocker and Entacopone 400mg, a peripheral catechol 0methyltransferase antagonist, and an additional 50mg dose of Carbidopa 30 minutes before 18 F-DOPA injection in order to provide increased availability of 18F-DOPA for striatal uptake. The purpose of the premedication regimen is to limit the metabolism of the 18 F-DOPA tracer by peripheral enzymes, i.e. DOPA decarboxylase and catechol O-methyltransferase, thereby maximizing central uptake (Koopmans et al.2007). Subjects will lay quietly on a gurney for 30 minutes before administration of the tracer to allow them to habituate into the environment and to relax. Subjects will then be injected with approximately $2.5 \mathrm{mCi}$ of 18F- DOPA. Scans will be acquired on a PET/CT Discovery 690 scanner. Dynamic scanning will start 90 minutes (Koopmans et al. 2007 ) after injection of tracer and continued for 100 minutes. $\underline{\text { fMRI data acquisition }}$

The fMRI data acquisition will take place at the Neuroradiology Department at the University Hospital Zurich. To measure brain structure and function, we use a 3.0 Tesla whole-body scanner. The measures include an anatomical scan, a diffusion MRI and a resting state functional MRI and a task based functional MRI. The total time in the scanner will be about 75 minutes. The subjects will perform the Fribourg reward task, an event related fMRI task adapted from the spatial delayed response task to measure neural responses to monetary reward (Gaillard et al. 2019).

\section{Ambulatory assessment measures and physiological measures}

To investigate the effects of the MORE intervention on daily affects, stress and reward experiences we will use ambulatory assessment (AA) measures. Primary outcomes related to the AA measures include 1) self-report measures of pain; 2) self-report measures and physiological measures (CAR, alpha-amylase awakening, and daily profile of cortisol and alpha-amylase) of stress, 3) measures of positive affect and 4) measures of reward experience. They will be compared before and after the MORE intervention between both FM groups. AA self-reports of pain, stress and reward and positive experience in daily life will be performed using an adaptation of the Experience Sampling Method (Wilhelm \& Schoebi, 2007) to assess the stress and reward experience in the daily living environment. The items related to pain are defined according to a pain diary that we have developed, tested and validated in 50 chronic pain patients at the University of Fribourg. The self-reported items measuring stress, positive and negative affect as well as reward experiences have additionally been adapted and validated in the framework of another research project (Guillod, 2017). All self-assessments are rated on a 7-point Likert scale. The participants will receive an Ipod and perform self-assessments during one week five times a day, during the week 
preceding or following the PET-measures before and after the MORE intervention. At the end of each day, participants randomly assigned to MORE will record the number of minutes spent engaged in the mindfulness, reappraisal, and savoring practices taught in the MORE intervention to provide a measure of adherence with the home practice associated with the MORE training. Biological measures of stress in everyday life will be assessed by collecting saliva samples 6 times a day ( 2 for the cortisol awakening response (CAR) and for the salivary alpha-amylase (AA) awakening, 4 for daily profile) for totally 3 days. Saliva samples will be obtained by using the passive drooling method (Salicap, IBL International, Hamburg, Germany). The CAR resp. AA awakening profile and the daily profiles will be used as biological indexes of stress reactivity in everyday life (Wilhelm \& Schoebi, 2007).

\section{Analysis plan}

\section{Primary outcome measures}

1. Effects of the MORE intervention on the DA response to monetary reward by comparing $18 \mathrm{~F}$ DOPA influx before and after the 8-week MORE intervention

\section{Secondary outcome measures:}

2. The percent BOLD signal change in striatal activity during the reward task before and after the 8-week MORE intervention

3. The correlation between daily practice of mindful breathing and savouring (minutes) and increased striatal activity and 18F DOPA influx

4. Changes in pain related outcomes after the MORE intervention

5. Changes in other clinical measures after the MORE intervention

6. Effects of the MORE intervention on daily affect, stress and reward experiences measured with AA measures

\section{Data analysis}

State of the art image processing techniques will be used to analyse the PET and the fMRI images. PET image pre-processing will be done using PMOD Version 4.1 or higher (PMOD software, PMOD technologies Zurich) and SPM (Wellcome Department of Imaging Neuroscience, London, UK) for the 18-F DOPA PET study. The most often used method to quantify biochemical function from 18-F DOPA PET image is the multiple time graphical approach (MTGA) that provides rate constants (Ki) for the storage of 18F- DOPA within regions of interest (ROIs) placed over the striatum (Patlak and Blaberg, 1995). Images from each dynamic DOPA PET dataset will be aligned and parametric images of 18F-DOPA influx (Ki) will be created for each subject. The Ki images will be transformed into standard stereotactic space. Regional $\mathrm{Ki}$ values of striatal regions (putamen, caudate, nucleus accumbens) will be analyzed with statistical parametric mapping for comparison of regional Ki values on a voxel by voxel basis. Group comparisons will be tested using independent samples T-test; treatment group $x$ time comparisons will be analysed 
with two factorial ANOVA's including Ki values obtained in the nucleus accumbens and the caudate to test the primary outcomes. The reward task will be performed in the fMRI scanner and we will correlate 18-FDopa influx with the striatal activation obtained with the fMRI task. In order to measure behavioural differences in the $\mathrm{fMRI}$ task results, in the ambulatory assessment data and in the intervention program's efficacy between the groups tested, we plan to use different kind of variance analyses and multilevel analyses. The number of participants for each part of the study has been determined in order to assure a good statistical power according with this specific research domain (e.g. including potential dropout, outliers). fMRI data analysis will be performed with state-of-the art software freely available online. T1 weighted scans will be analysed with regard to cortical thickness and subcortical volumes using surface based morphometry implemented in FreeSurfer software suite 5.0.1.(http://surfer.nmr.mgh.harvard.edu). This includes a fully automated method. Briefly, this processing includes 1) motion correction, 2) removal of non-brain tissue, 3) automated Talairach transformation, 4) segmentation of the subcortical white matter and deep gray matter volumetric structures (amygdala, hippocampus, thalamus, caudate, putamen, pallidum, nucleus accumbens, ventricles), 5) intensity normalization, 6) tessellation of the gray matter/white matter boundary, 7) automated topology correction, and 8) surface deformation following intensity gradients to optimally place the gray/white and gray/cerebrospinal fluid borders at the location where the greatest shift in intensity defines the transition to the other tissue class. Freesurfer morphometric procedures have been demonstrated to show good test-retest reliability across scanner manufacturers and across field strengths. Obtained subcortical volumes will be normalized by individual intra-cranial volumes for further statistical analyses. Exploratory approaches will involve a vertex-based analysis across the whole brain. Diffusion MRI scans will be analysed for structural connectivity with FMRIB software library 4.1.9 (FSL, http://www.fmrib.ox.ac.uk/fsl). Functional MRI scans will be analysed for activity and functional connectivity with the latest version of Statistical Parametric Mapping (SPM, http://www.fil.ion.ucl.ac.uk/spm). Functional MRI data will be pre-processed according to the following steps: 1) slice timing correction, 2) realignment, 3) linear and non-linear normalization on to a standard EPI template, 4) voxel re-sampling to $2 \times 2 \times 2 \mathrm{~mm}^{3} 5$ ) smoothing with a Gaussian kernel of $6 \mathrm{~mm}$ full width at half maximum, 6) detrending, 7) filtering (such that frequencies $0.01<f<0.08 \mathrm{~Hz}$ passed the filter), and 8 ) regressing out the variance of nuisance covariates. With REST toolbox 1.6, for each subject and each specified region of interest (atlas-based specification), mean signal time courses will be extracted and cross-correlated. Next, correlations will be r-to-z transformed for group-level statistics. These z-values can be used for comparisons across (cross-sectional) and within (longitudinal) groups or for parametric correlations with psychometric measures. The main analyses of the measures related to the primary outcomes of the clinical effects of MORE, including measures of pain intensity and severity, measures of functioning, measures of sleep, and measures of mood outcomes will be performed with two-factorial (time $x$ treatment group) ANCOVA controlling for pain score intensity at pre-test according to (Garland et al., 2014) as well as one ANCOVA controlling for depressive symptoms at pre-test. Measures related to the primary outcomes of the clinical effects of MORE, including measures of pain intensity and severity, measures of functioning, measures of sleep, and measures of mood will be performed with ANOVA's. In case of not normally distributed data, we will use a transformation in Z-scores, rather than using nonparametric tests. We will perform per protocol analyses to test our hypotheses. Intent-to-treat analyses 
will be used for sensitivity analyses (Garland et al., 2014). To account for missing data we will use multiple imputation procedures.

Analyses of the secondary outcomes related to the treatment mechanisms will be performed using twofactorial (treatment group $x$ time) ANOVAs to test the pre-post-test differences. The AA measures yield intensive longitudinal data that are clustered, as they represent series of measurements that stem from different individuals. A multilevel approach to analyse these data takes into account clustering and can therefore accommodate these data and provides flexible tools to investigate within-subject phenomena, such as responses to stressors or rewarding experiences (see Bolger \& Laurenceau, 2013). We will use a software that allows for the simultaneous modelling of within-subject and between-subject aspects of the data, and the examination of associations among individual difference variables, and individual differences in within-subject parameters (e.g., Mplus 7.3, (Muthen \& Muthen, 1998-2012). The sample size of this study is based on: 1) our previous research (Ledermann et al. 2016) that showed large effect sizes (Cohen's $d 1.1 ; F=1.3)$ in the comparison between our healthy controls $(N=17)$ and FM participants without depression ( $N=17)$ for the differences in DA binding in response to unpredictable reward. 2) Earlier studies investigating neurophysiological changes in MORE with EEG that reported significant changes $\left(F_{1,25}=4.9 ;\right.$ npartial $\left.{ }^{2}=.17\right)$ in response to reward before and after the MORE intervention in chronic pain patients with sample sizes of $\mathrm{N}=11$ (active group) and $\mathrm{N}=18$ (support group). Using this effect size, a power analysis with G*Power (Faul et al. 2007) showed that 46 participants are necessary for an ANOVA with 2 factors and repeated measures to be significant. Compared to Garland et al. 2015 we will use a waiting-list design in this study and not an active intervention as control, what might increase the effect size of the results. In addition, results obtained with EEG cannot be directly compared to PET measures. For these reasons, we interpolate the sample size between the different estimations obtained that the sample size after the intervention should be between $\mathrm{N}=12$ based on Backman et al. 2011 and $N=46$ based on Garland et al. 2015, taking into account that a strict minimum of participants should be exposed to radioactivity for the PET measures. With regard to the clinical measures, the RCT by Garland et al. 2014 evidenced significant reductions in pain severity with medium effect size (Cohen's d = 0.5) after the MORE intervention in a starting group of 115 participants and of 69 at post-intervention measures ( $\mathrm{N}=31$ for MORE and $\mathrm{N}=38$ for support group). Based on these estimates, an ANCOVA to be significant should have a minimal sample of $\mathrm{N}=25$ participants after treatment in each group. Finally, with regard to the daily measures of positive affect, pain and stress, Garland et al. 2017 found significant effects associated with the MORE intervention compared to a support group therapy in a group of $\mathrm{N}=55$ (MORE. $N=26$, Support Group: $N=29$ ) using multilevel analyses. In order to be able to do group comparisons with the AA measures, we will add 15 healthy participants who will only do the AA part. On the basis of these analyses, we consider that an estimation of 30 participant in each condition (waitinglist versus MORE) after treatment should be sufficient to obtain significant results for our aims. In their randomized clinical trial of the MORE intervention in chronic pain patients with opioid abuse, Garland et al. 2014 found $20 \%$ drop-out between beginning and completion of the intervention. We will therefore postulate $20 \%$ drop-out in total, giving a starting sample of 38 participants in each group, that we will round up to 40 to account for data loss related to technical problems, giving a total sample of 80 .

Page $14 / 29$ 


\section{Discussion}

Among chronic pain conditions, FM is a frequent and very disabling disease, which is still poorly understood and difficult to treat. This project aims to investigate if the mindfulness based MORE intervention is able to restore the DA function in FM patients, in particular with regard to DA responses to reward, and to reduce pain and mood symptoms. More specifically, we expect the FM participants to show altered DA responses to reward before MORE compared to healthy controls; and we expect these alterations to be reduced after the MORE intervention compared to a wait-list group. Building on a previous project of our group pointing to a dysfunction of the DA system in FM (Ledermann et al. 2017), we will extend here the understanding of the role of DA in FM and investigate the potential of a nonpharmacological intervention to decrease pain and ameliorate mood in FM as well as to induce changes in the central DA function. With regard to the clinical effects of MORE, Garland et al. 2014 found medium effect size of the intervention on pain severity. We used their effect size to calculate the size of our sample for the primary outcome of DA responses to monetary reward and are confident that the use of a multi-modal design combining clinical and AA measures will permit a better detection of the effects. At a clinical level, the use of standardized outcomes recommended for the study of FM will allow us to target clear variables for changes associated with the intervention. By using a wait-list design instead of a design with an active control group might increase the effects as we will not control for placebo effects. However, the justification is based on ethical and methodological reflexions that have been discussed in the design section, and constitute for this sample with these specific measures are the state-of-the-art. We are aware that proper AA analyses need larger samples, however the sample sizes calculated correspond to the main outcome and other studies in Fibromyalgia studies and will allow for exploratory microanalyses.

At a scientific level, this project is highly innovative as it will integrate in vivo brain imaging of the DA system in response to motivational stimuli with AA measures in everyday life, allowing for association between daily life momentary affective states and self-reported reward sensitivity with DA transmission elicited by rewards in an experimental setting. In addition, the investigation of potential changes of the DA reactivity after a mindfulness-based intervention reveal crucial information concerning the potential plasticity of the DA system. At a clinical level, MORE is a manualized therapy comparably easy to teach and implement. It could therefore be used in several clinical settings and at a larger scale. The integration of a large network of outpatient clinics in this project will allow for a rapid dissemination of the methods if MORE shows significant results. The integration of AA measures as outcome measures for the MORE intervention is not only innovative, but it will also allow a transfer of the intervention-related training effects in everyday life of the participants so to increase the ecological validity of our results. Finally, we are confident that our results will both bring a better understanding of FM as well as integrating neuroscience findings into treatment development by targeting neural mechanisms underlying FM. However, some challenges need to be addressed. We have the following reasons to justify the use of a design with a waiting-list group and not an active control group. First, placebo group interventions in the psychological setting, often lacking credibility can elicit so-called "resentful demoralization" Schwartz et al. 1997, leading to increased drop-out risk in these groups. With regard to the study of Garland 2014 
indicating more than $20 \%$ drop-out during the treatment, it is important to minimize this risk. From an ethical point of view, it is questionable to provide a non-efficacious treatment to patients having a long history of such treatments as it is unfortunately the experience of many FM patients. Because of the symptomatology (mobility difficulties due to pain symptoms, fatigue etc.), it is difficult for patients to commit to a treatment, so it would be a waste of both time and energy for them to come to our centers for pseudo-intervention. From a methodological point-of-view, support group interventions are problematic: they will not be comparable across time and different groups since the interaction between the participants and the therapists cannot be fully standardized. This is particularly complicated when the intervention is given in 3 different centers, and might impair the validity of such a control intervention. For these reasons, RCT list designs can be justified to assess the effects of psychological interventions in populations with somatic disorders. To minimize bias, we will use only standardized questionnaires to assess the secondary outcomes as recommended by the international organized network aiming at improving outcome measures in rheumatology (OMERACT). Recruitment in general is a challenge for large clinical trials with chronic pain patients, but we have faced this challenge previously and have developed effective strategies for recruitment (Ledermann et al. 2016, Ledermann et al, 2017). For this more ambitious trial. Recruiting multi-centrically participants in the German and the French speaking parts of Switzerland will allow to complete the study. In addition, we have created a large network of outpatient clinics and practices with our project partners at the different centers, who will directly support the study with recruitment. With regard to the medication, opioids should not be too much of a concern, given low efficacy in these patients, and relatively restrictive prescription practices in Switzerland for this population.

Another barrier of recruitment is going to be the requirement of randomization into immediate or delayed groups. As such, participants will have to make themselves available for program dates over a period of 5 months, in case they are randomized to the waitlist groups. Logistically managing simultaneous baseline and follow-up measures can be challenging.

Another issue is drop-outs or missing data. By using an online format for the questionnaires, we aim to eliminate incomplete datasets. Due to the forced-answer format of the questions in the questionnaires, no missing data is to be expected. In case of missing data, the last observation will be carried forward. Dropouts will be replaced in order to reach the targeted number of complete participants in the study. To evaluate the influence of dropouts, analyses will be first performed with complete datasets only, and compared to results of intent-to-treat analyses where all participants are included.

\section{Trial Status}

The protocol number version 6, dated 31.03.2021. Recruitment started in June 2021 and will approximately be finished in October 2024. The protocol was registered under ClinicalTrials.gov NCT 044515664. Registered on 3 July 2020. The trial was prospectively registered.

\section{Declarations}




\section{Funding}

This study will be supported by the Swiss National Foundation Grant Number 325130_182766. The role of the funding body did not influence the design of the study and will also not influence collection, analysis and interpretation of the data in the manuscript.

\section{Ethics approval and consent to participate}

Ethics approval was taken from the Ethics Committee (CEC) of Zurich and Vaud. Ethics approval was given on June $1^{\text {st }} 2021$ (2020-00045). Substantial protocol amendments (significant changes) are only implemented after approval of the CEC. Under emergency circumstances, deviations from the protocol to protect the rights, safety and well-being of human participants may proceed without prior approval of the sponsor and the CEC. Such deviations shall be documented and reported to the sponsor and the CEC as soon as possible. A list of substantial amendments is also available on www.swissethics.ch.

All participants for this study will be provided a participant information sheet and a consent form describing this study and providing sufficient information for participants to make an informed decision about their participation in this study.

The participant information sheet and the consent form was submitted with the protocol for review and approval for the study by the CEC. The formal consent of a participant, using the approved consent form, will be obtained before that participant is submitted to any study procedure.

\section{Confidentiality:}

The investigators are liable to treat the entire information related to the study and the compiled data strictly confidentially. Any passing-on of information to persons that are not directly involved in the study must be approved by the owner of the information.

Data generation, transmission, archiving and analysis of personal data within this study, strictly follows the current Swiss legal requirements for data protection. Prerequisite is the voluntary approval of the Participant given by signing the informed consent prior start of participation of the clinical trial.

Individual participant medical information obtained as a result of this study is considered confidential and disclosure to third parties is prohibited. Participant's confidentiality will be further ensured by utilizing participant identification code numbers to correspond to treatment data in the computer files. Such medical information may be given to the participant's personal physician or to other appropriate medical personnel responsible for the participant's welfare, if the patient has given his/her written consent to do so. Data generated as a result of this study are to be available for inspection on request by the monitors and by the $\mathrm{CEC}$.

\section{Data monitoring}


Monitoring visits at the investigator's site prior to the start and during the course of the study will help to follow up the progress of the clinical study, to assure utmost accuracy of the data and to detect possible errors at an early time point. The Sponsor organizes professional independent monitoring for the study. All original data including all patient files, progress notes and copies of laboratory and medical test results will be available for monitoring. The monitor will review all or a part of the eCRFs and written informed consents. The accuracy of the data will be verified by reviewing the above referenced documents. The investigator's site will collaborate with the Appletree $\mathrm{Cl}$ group.

\section{Data monitoring: Interim analysis}

Interim analyses will include pre-test comparisons (healthy controls versus FMS) on the main and secondary outcomes after completion of the first MORE group as well as exploratory pre-post comparisons on the main outcomes to control whether methodological adjustments have to be made. Data of the pre-test comparisons (healthy controls versus FMS) after completion of the second MORE group will be used for first data presentation and if possible publication of pilot data. The sponsorInvestigator can make the final decision to terminate the trial.

\section{Harms:}

During the entire duration of the study, all serious and non-serious adverse events (SAEs) that may be causally related to the study intervention are collected and documented in source documents. Reportable events are recorded in the case report form (CRF). Study duration encompassed the time from when the participant signs the informed consent until the last protocol-specific procedure has been completed, including a safety follow-up period of 12 weeks.

The recording of serious and non-serious adverse event (SAE and AE) information includes time of onset, duration, resolution, action to be taken, assessment of intensity, relationship with study treatment. Participants will be asked about health problems, mood worsening and negative thoughts as well as any emergency medical or hospitalization that could have happened between 2 study visits at each visit, beginning at pre-test. Participants who prematurely stop the study will be asked for adverse events at the time, when they stop at the end of study visit.

\section{Auditing:}

A quality assurance audit/inspection of this study may be conducted by the CEC. The quality assurance auditor/inspector will have access to all medical records, the investigator's study related files and correspondence, and the informed consent documentation that is relevant to this clinical study.

The investigator will allow the persons being responsible for the audit or the inspection to have access to the source data/documents and to answer any questions arising. All involved parties will keep the patient data strictly confidential.

\section{Dissemination:}


Trial results:

After the statistical analysis of this trial the sponsor will make every endeavor to publish the data in a peer-reviewed medical or psychological journal. In addition, preliminary data (from interim analyses) will be presented in scientific meetings and if possible published.

Authorship:

Authorship for all investigators and associated investigators, no intended use of professional writers.

The trial results will be published as peer-reviewed scientific papers and poster or oral presentations in conferences. All data and protocol will be available beginning three months and ending three years after publication of the results. The trial data will be available from the corresponding author upon reasonable request.

\section{Availability of data and materials}

The trial results will be published as peer-reviewed scientific papers and poster or oral presentations in conferences. All data and protocol will be available beginning three months and ending three years after publication of the results. The trial data will be available from the corresponding author upon reasonable request.

\section{Author's contributions}

The trial was designed and developed by CMS, and KL. The manuscript was drafted by KL. The protocol was carefully revised and edited by RvK, CB, HS, JJ and EG. All authors read and approved the final manuscript.

\section{Competing interests}

The authors declare that they have no competing interests.

\section{References}

1. Aman M, Jason Y, Kaye A, Urman R. (2018). Evidence-Based Non-Pharmacological Therapies for Fibromyalgia. Curr Pain Headache Rep. 2018;22(5):33. Published 2018 Apr 4. doi:10.1007/s11916018-0688-2.

2. Backman L, Nyberg L, Soveri A, Johansson J, Andersson M, Dahlin E,.. . Rinne JO. Effects of workingmemory training on striatal dopamine release. Science. 2011;333(6043):718. doi:10.1126/science.1204978.

3. Baer RA, Smith GT, Hopkins J, Krietemeyer J, Toney L. Using self-report assessment methods to explore facets of mindfulness. Assessment. 2006;13(1):27-45. doi:10.1177/1073191105283504. 
4. Baliki MN, Apkarian AV. Nociception, Pain, Negative Moods, and Behavior Selection. Neuron. 2015;87(3):474-91. doi:10.1016/j.neuron.2015.06.005.

5. Baliki MN, Geha PY, Fields HL, Apkarian AV. Predicting value of pain and analgesia: nucleus accumbens response to noxious stimuli changes in the presence of chronic pain. Neuron. 2010;66(1):149-60. doi:10.1016/j.neuron.2010.03.002.

6. Beck AT, Ward CH, Mendelson M, Mock J, Erbaugh J. An inventory for measuring depression. Arch Gen Psychiatry. 1961;4:561-71.

7. Bellato E, Marini E, Castoldi F, Barbasetti N, Mattei L, Bonasia DE, Blonna D. (2012). Fibromyalgia syndrome: etiology, pathogenesis, diagnosis, and treatment. Pain Res Treat, 2012, 426130. doi:10.1155/2012/426130.

8. Bennett RM, Friend R, Jones KD, Ward R, Han BK, Ross RL. The Revised Fibromyalgia Impact Questionnaire (FIQR): validation and psychometric properties. Arthritis Res Ther. 2009;11(4):R120. doi:10.1186/ar2783.

9. Bolger N, Laurenceau JP. Intensive longitudinal methods: An introduction to diary and experience sampling research. New York: Guilford Press; 2013.

10. Brooks P, Boers M, Simon LS, Strand V, Tugwell P, Idzerda L. (2011). OMERACT 10-International Consensus Conference on Outcome Measures in Rheumatology Clinical Trials. J Rheumatol, 38(7), 1450-1451. doi:10.3899/jrheum.110325.

11. Brown GK, Nicassio PM. Development of a questionnaire for the assessment of active and passive coping strategies in chronic pain patients. Pain. 1987;31(1):53-64.

12. Bryant F. Savoring Beliefs Inventory (SBI): A scale for measuring beliefs about savoring. Journal of Mental Health. 2003;12(2):175-96.

13. Bushnell MC, Ceko M, Low LA. Cognitive and emotional control of pain and its disruption in chronic pain. Nat Rev Neurosci. 2013;14(7):502-11. doi:10.1038/nrn3516.

14. Cleeland CS, Ryan KM. Pain assessment: global use of the Brief Pain Inventory. Ann Acad Med Singapore. 1994;23(2):129-38.

15. Cohn MA, Fredrickson BL, Brown SL, Mikels JA, Conway AM. Happiness unpacked: positive emotions increase life satisfaction by building resilience. Emotion. 2009;9(3):361-8. doi:10.1037/a0015952.

16. Crofford LJ, Appleton BE. The treatment of fibromyalgia: a review of clinical trials. Curr Rheumatol Rep. 2000;2(2):101-3.

17. de Jong M, Peeters F, Gard T, Ashih H, Doorley J, Walker R,.. . Mischoulon D. A Randomized Controlled Pilot Study on Mindfulness-Based Cognitive Therapy for Unipolar Depression in Patients With Chronic Pain. J Clin Psychiatry. 2017. doi:10.4088/JCP.15m10160.

18. Delespaul PA, deVries MW. The daily life of ambulatory chronic mental patients. J Nerv Ment Dis. 1987;175(9):537-44.

19. Dillmann U, Nilges $P$, Saile H, Gerbershagen HU. [Assessing disability in chronic pain patients.]. Schmerz. 1994;8(2):100-10. doi:10.1007/BF02530415. 
20. Drevets WC, Gautier C, Price JC, Kupfer DJ, Kinahan PE, Grace AA,.. . Mathis CA. Amphetamineinduced dopamine release in human ventral striatum correlates with euphoria. Biol Psychiatry. 2001;49(2):81-96.

21. Drevets WC, Price JC, Kupfer DJ, Kinahan PE, Lopresti B, Holt D, Mathis C. PET measures of amphetamine-induced dopamine release in ventral versus dorsal striatum. Neuropsychopharmacology. 1999;21(6):694-709. doi:10.1016/S0893-133X(99)00079-2.

22. Faul F, Erdfelder E, Lang AG, Buchner A. G*Power 3: a flexible statistical power analysis program for the social, behavioral, and biomedical sciences. Behav Res Methods. 2007;39(2):175-91.

23. Finan PH, Smith MT. The comorbidity of insomnia, chronic pain, and depression: dopamine as a putative mechanism. Sleep Med Rev. 2013;17(3):173-83. doi:10.1016/j.smrv.2012.03.003.

24. Finan PH, Zautra AJ, Davis MC. Daily affect relations in fibromyalgia patients reveal positive affective disturbance. Psychosom Med. 2009;71(4):474-82. doi:10.1097/PSY.0b013e31819e0a8b.

25. Fischer S, Doerr JM, Strahler J, Mewes R, Thieme K, Nater UM. Stress exacerbates pain in the everyday lives of women with fibromyalgia syndrome-The role of cortisol and alpha-amylase. Psychoneuroendocrinology. 2016;63:68-77. doi:10.1016/j.psyneuen.2015.09.018.

26. Froeliger B, Mathew AR, McConnell PA, et al. Restructuring Reward Mechanisms in Nicotine Addiction: A Pilot fMRI Study of Mindfulness-Oriented Recovery Enhancement for Cigarette Smokers. Evid Based Complement Alternat Med. 2017;2017:7018014. doi:10.1155/2017/7018014.

27. Gaillard C, Guillod M, Ernst M, Torrisi S, Federspiel A, Schoebi D, ... Homan P. Striatal responsiveness to reward under threat-of-shock and working memory load: A preliminary study. Brain behavior. 2019;9(10):e01397.

28. Garland EL, Roberts-Lewis A, Tronnier CD, Graves R, Kelley K. Mindfulness-Oriented Recovery Enhancement versus CBT for co-occurring substance dependence, traumatic stress, and psychiatric disorders: Proximal outcomes from a pragmatic randomized trial [published correction appears in Behav Res Ther. 2018 Jan;100:78]. Behav Res Ther. 2016;77:7-16. doi:10.1016/j.brat.2015.11.012.

29. Garland EL, Bryan CJ, Finan PH, Thomas EA, Priddy SE, Riquino MR, Howard MO. Pain, hedonic regulation, and opioid misuse: Modulation of momentary experience by Mindfulness-Oriented Recovery Enhancement in opioid-treated chronic pain patients. Drug Alcohol Depend. 2017;173(Suppl 1):65-72. doi:10.1016/j.drugalcdep.2016.07.033.

30. Garland EL, Bryan CJ, Finan PH, et al. Pain, hedonic regulation, and opioid misuse: Modulation of momentary experience by Mindfulness-Oriented Recovery Enhancement in opioid-treated chronic pain patients. Drug Alcohol Depend. 2017;173(Suppl 1):65-72.

doi:10.1016/j.drugalcdep.2016.07.033.

31. Garland EL, Froeliger B, Howard MO. Effects of Mindfulness-Oriented Recovery Enhancement on reward responsiveness and opioid cue-reactivity. Psychopharmacology. 2014;231(16):3229-38. doi:10.1007/s00213-014-3504-7.

32. Garland EL, Froeliger B, Howard MO. Neurophysiological evidence for remediation of reward processing deficits in chronic pain and opioid misuse following treatment with Mindfulness-Oriented 
Recovery Enhancement: exploratory ERP findings from a pilot RCT. J Behav Med. 2015;38(2):32736. doi:10.1007/s10865-014-9607-0.

33. Garland EL, Froeliger B, Zeidan F, Partin K, Howard MO. The downward spiral of chronic pain, prescription opioid misuse, and addiction: cognitive, affective, and neuropsychopharmacologic pathways. Neurosci Biobehav Rev. 2013;37(10 Pt 2):2597-607.

doi:10.1016/j.neubiorev.2013.08.006.

34. Garland EL, Gaylord SA, Boettiger CA, Howard MO. Mindfulness training modifies cognitive, affective, and physiological mechanisms implicated in alcohol dependence: results of a randomized controlled pilot trial. J Psychoactive Drugs. 2010;42(2):177-92. doi:10.1080/02791072.2010.10400690.

35. Garland EL, Howard MO, Zubieta JK, Froeliger B. Restructuring Hedonic Dysregulation in Chronic Pain and Prescription Opioid Misuse: Effects of Mindfulness-Oriented Recovery Enhancement on Responsiveness to Drug Cues and Natural Rewards. Psychother Psychosom. 2017;86(2):111-2. doi:10.1159/000453400.

36. Garland EL, Manusov EG, Froeliger B, Kelly A, Williams JM, Howard MO. Mindfulness-oriented recovery enhancement for chronic pain and prescription opioid misuse: results from an early-stage randomized controlled trial. J Consult Clin Psychol. 2014;82(3):448-59. doi:10.1037/a0035798.

37. Garland EL, Hanley AW, Riquino MR, et al. Mindfulness-oriented recovery enhancement reduces opioid misuse risk via analgesic and positive psychological mechanisms: A randomized controlled trial. J Consult Clin Psychol. 2019;87(10):927-40. doi:10.1037/ccp0000390.

38. Garland EL, Hanley AW, Kline A, Cooperman NA. Mindfulness-Oriented Recovery Enhancement reduces opioid craving among individuals with opioid use disorder and chronic pain in medication assisted treatment: Ecological momentary assessments from a stage 1 randomized controlled trial. Drug Alcohol Depend. 2019;203:61-5. doi:10.1016/j.drugalcdep.2019.07.007.

39. Garland EL, Atchley RM, Hanley AW, Zubieta JK, Froeliger B. Mindfulness-Oriented Recovery Enhancement remediates hedonic dysregulation in opioid users: Neural and affective evidence of target engagement. Sci Adv. 2019;5(10):eaax1569. Published 2019 Oct 16. doi:10.1126/sciadv.aax1569.

40. Garnefski N, Rieffe C, Jellesma F, Terwogt MM, Kraaij V. Cognitive emotion regulation strategies and emotional problems in 9-11-year-old children: the development of an instrument. Eur Child Adolesc Psychiatry. 2007;16(1):1-9. doi:10.1007/s00787-006-0562-3.

41. Geschwind N, Peeters F, Jacobs N, Delespaul P, Derom C, Thiery E,.. . Wichers M. Meeting risk with resilience: high daily life reward experience preserves mental health. Acta Psychiatr Scand. 2010;122(2):129-38. doi:10.1111/j.1600-0447.2009.01525.x.

42. Glombiewski JA, Sawyer AT, Gutermann J, Koenig K, Rief W, Hofmann SG. Psychological treatments for fibromyalgia: a meta-analysis. Pain. 2010;151(2):280-95. doi:10.1016/j.pain.2010.06.011.

43. Goldenberg DL, Burckhardt C, Crofford L. Management of fibromyalgia syndrome. JAMA. 2004;292(19):2388-95. doi:10.1001/jama.292.19.2388. 
44. Goyal M, Singh S, Sibinga EM, et al. Meditation programs for psychological stress and well-being: a systematic review and meta-analysis. JAMA Intern Med. 2014;174(3):357-68. doi:10.1001/jamainternmed.2013.13018.

45. Grossman P, Niemann L, Schmidt S, Walach H. Mindfulness-based stress reduction and health benefits. A meta-analysis. J Psychosom Res. 2004;57(1):35-43. doi:10.1016/S0022-3999(03)005737.

46. Guillod M, Gaillard C, Recabarren R, Schoebi D, Merlo M, Hasler G, Martin-Soelch C. (2017). Measures of daily mood, stress and reward responses in offspring of depressed parents. Paper presented at the 13th Congress of the World Congress on Biological Psychiatry, Copenhagen.

47. Hagelberg N, Forssell H, Aalto S, Rinne JO, Scheinin H, Taiminen T,... Jaaskelainen SK. Altered dopamine D2 receptor binding in atypical facial pain. Pain. 2003;106(1-2):43-8.

48. Hagelberg N, Forssell H, Rinne JO, Scheinin H, Taiminen T, Aalto S,... Jaaskelainen S. Striatal dopamine D1 and D2 receptors in burning mouth syndrome. Pain. 2003;101(1-2):149-54.

49. Hamilton M. A rating scale for depression. J Neurol Neurosurg Psychiatry. 1960;23:56-62.

50. Hashmi JA, Baliki MN, Huang L, Baria AT, Torbey S, Hermann KM,.. . Apkarian AV. Shape shifting pain: chronification of back pain shifts brain representation from nociceptive to emotional circuits. Brain. 2013;136(Pt 9):2751-68. doi:10.1093/brain/awt211.

51. Hauser W, Wolfe F. Diagnosis and diagnostic tests for fibromyalgia (syndrome). Reumatismo. 2012;64(4):194-205. doi:10.4081/reumatismo.2012.194.

52. Hautzinger M. [The Beck Depression Inventory in clinical practice]. Nervenarzt. 1991;62(11):689-96.

53. Hays RD, Stewart AL. Sleep measures. In: Stewart AL, Ware JE, Jr, editors. Measuring functioning and weel-being: the Medical Outcomes Study Approach. Durham (NC): Duke University Press; 1992. pp. 235-59.

54. Hipolito L, Wilson-Poe A, Campos-Jurado Y, Zhong E, Gonzalez-Romero J, Virag L,... Moron JA. Inflammatory Pain Promotes Increased Opioid Self-Administration: Role of Dysregulated Ventral Tegmental Area mu Opioid Receptors. J Neurosci. 2015;35(35):12217-31. doi:10.1523/JNEUROSCI.1053-15.2015.

55. Jones GT, Atzeni F, Beasley M, Fluss E, Sarzi-Puttini P, Macfarlane GJ. The prevalence of fibromyalgia in the general population: a comparison of the american college of rheumatology 1990, 2010, and modified 2010 classification criteria. Arthritis Rheumatol. 2015;67(2):568-75. doi:10.1002/art.38905.

56. Kahl KG, Winter L, Schweiger U. The third wave of cognitive behavioural therapies: what is new and what is effective? Curr Opin Psychiatry. 2012;25(6):522-8. doi:10.1097/YC0.0b013e328358e531.

57. Kia S, Choy E. (2017). Update on Treatment Guideline in Fibromyalgia Syndrome with Focus on Pharmacology. Biomedicines, 5(2). doi:10.3390/biomedicines5020020.

58. Laux LG, P. (1981). Das State Trait Angstinventar, Weinheim, Beltz. 
59. Ledermann K, Jenewein J, Sprott H, Hasler G, Schnyder U, Warnock G,.. . Martin-Soelch C. (2017). Altered dopamine responses to monetary rewards in female fibromyalgia patients with and without depression: a [11C] raclopride bolus-plus-infusion PET study. Psychotherapy and psychosomatics, In press.

60. Ledermann K, Jenewein J, Sprott H, Hasler G, Schnyder U, Warnock G,.. . Martin-Soelch C. Relation of dopamine receptor 2 binding to pain perception in female fibromyalgia patients with and without depression-A [(1)(1)C] raclopride PET-study. Eur Neuropsychopharmacol. 2016;26(2):320-30. doi:10.1016/j.euroneuro.2015.12.007.

61. Lee YH, Song GG. Comparative efficacy and tolerability of duloxetine, pregabalin, and milnacipran for the treatment of fibromyalgia: a Bayesian network meta-analysis of randomized controlled trials. Rheumatol Int. 2016;36(5):663-72. doi:10.1007/s00296-016-3468-5.

62. Loggia ML, Berna C, Kim J, Cahalan CM, Gollub RL, Wasan AD,.. . Napadow V. Disrupted brain circuitry for pain-related reward/punishment in fibromyalgia. Arthritis Rheumatol. 2014;66(1):20312. doi:10.1002/art.38191.

63. Martin-Soelch C. Is depression associated with dysfunction of the central reward system? Biochem Soc Trans. 2009;37(Pt 1):313-7. doi:10.1042/BST0370313.

64. Martin-Soelch C, Szczepanik J, Nugent A, Barhaghi K, Rallis D, Herscovitch P,.. . Drevets WC. Lateralization and gender differences in the dopaminergic response to unpredictable reward in the human ventral striatum. Eur J Neurosci. 2011;33(9):1706-15. doi:10.1111/j.14609568.2011.07642.x.

65. Muthén LK, Muthén BO. ((1998-2012)). Mplus User's Guide. Seventh Edition. Los Angeles, CA: Muthén \& Muthén.

66. Niikura K, Narita M, Butelman ER, Kreek MJ, Suzuki T. Neuropathic and chronic pain stimuli downregulate central mu-opioid and dopaminergic transmission. Trends Pharmacol Sci. 2010;31(7):299-305. doi:10.1016/j.tips.2010.04.003.

67. O'Doherty JP. Reward representations and reward-related learning in the human brain: insights from neuroimaging. Curr Opin Neurobiol. 2004;14(6):769-76. doi:10.1016/j.conb.2004.10.016.

68. Oldfield RC. The assessment and analysis of handedness: The Edinburgh inventory. Neuropsychologia. 1971;9(1):97-113.

69. Papadopoulou D, Fassoulaki A, Tsoulas C, Siafaka I, Vadalouca A. A meta-analysis to determine the effect of pharmacological and non-pharmacological treatments on fibromyalgia symptoms comprising OMERACT-10 response criteria. Clin Rheumatol. 2016;35(3):573-86. doi:10.1007/s10067-015-3144-2.

70. Penrod JR, Bernatsky S, Adam V, Baron M, Dayan N, Dobkin PL. Health services costs and their determinants in women with fibromyalgia. J Rheumatol. 2004;31(7):1391-8.

71. Potvin S, Grignon S, Marchand S. Human evidence of a supra-spinal modulating role of dopamine on pain perception. Synapse. 2009;63(5):390-402. doi:10.1002/syn.20616. 
72. Rosenzweig S, Greeson JM, Reibel DK, Green JS, Jasser SA, Beasley D. Mindfulness-based stress reduction for chronic pain conditions: variation in treatment outcomes and role of home meditation practice. J Psychosom Res. 2010;68(1):29-36. doi:10.1016/j.jpsychores.2009.03.010.

73. Rossy LA, Buckelew SP, Dorr N, Hagglund KJ, Thayer JF, McIntosh MJ,.. . Johnson JC. A metaanalysis of fibromyalgia treatment interventions. Ann Behav Med. 1999;21(2):180-91.

74. Schwartz CE, Chesney MA, Irvine MJ, Keefe FJ. The control group dilemma in clinical research: applications for psychosocial and behavioral medicine trials. Psychosom Med. 1997;59(4):362-71.

75. Segal Z, Vincent P, Levitt A. Efficacy of combined, sequential and crossover psychotherapy and pharmacotherapy in improving outcomes in depression. J Psychiatry Neurosci. 2002;27(4):281-90.

76. Sheehan DV, Lecrubier Y, Sheehan KH, Amorim P, Janavs J, Weiller E,.. . Dunbar GC. (1998). The MiniInternational Neuropsychiatric Interview (M.I.N.I.): the development and validation of a structured diagnostic psychiatric interview for DSM-IV and ICD-10. J Clin Psychiatry, 59 Supp/ 20, 22-33;quiz 34-57.

77. Sim J, Adams N. Systematic review of randomized controlled trials of nonpharmacological interventions for fibromyalgia. Clin J Pain. 2002;18(5):324-36.

78. Skevington SM, Lotfy M, O'Connell KA, Group W. The World Health Organization's WHOQOL-BREF quality of life assessment: psychometric properties and results of the international field trial. A report from the WHOQOL group. Qual Life Res. 2004;13(2):299-310. doi:10.1023/B:QURE.0000018486.91360.00.

79. Stewart AL, Hays RD, Ware JE Jr. The MOS short-form general health survey. Reliability and validity in a patient population. Med Care. 1988;26(7):724-35.

80. Taylor AM, Becker S, Schweinhardt P, Cahill C. Mesolimbic dopamine signaling in acute and chronic pain: implications for motivation, analgesia, and addiction. Pain. 2016;157(6):1194-8. doi:10.1097/j.pain.0000000000000494.

81. Theadom A, Cropley M, Smith HE, Feigin VL, McPherson K. Mind and body therapy for fibromyalgia. Cochrane Database Syst Rev. 2015;4:CD001980. doi:10.1002/14651858.CD001980.pub3.

82. Thieme K, Mathys M, Turk DC. Evidenced-Based Guidelines on the Treatment of Fibromyalgia Patients: Are They Consistent and If Not, Why Not? Have Effective Psychological Treatments Been Overlooked? J Pain. 2016. doi:10.1016/j.jpain.2016.12.006.

83. Thieme K, Mathys M, Turk DC. Evidenced-Based Guidelines on the Treatment of Fibromyalgia Patients: Are They Consistent and If Not, Why Not? Have Effective Psychological Treatments Been Overlooked? J Pain. 2017;18(7):747-56. doi:10.1016/j.jpain.2016.12.006.

84. Treede RD, Jensen TS, Campbell JN, Cruccu G, Dostrovsky JO, Griffin JW... . Serra J. Neuropathic pain: redefinition and a grading system for clinical and research purposes. Neurology. 2008;70(18):1630-5. doi:10.1212/01.wnl.0000282763.29778.59.

85. Veehof MM, Trompetter HR, Bohlmeijer ET, Schreurs KM. Acceptance- and mindfulness-based interventions for the treatment of chronic pain: a meta-analytic review. Cogn Behav Ther. 2016;45(1):5-31. doi:10.1080/16506073.2015.1098724. 
86. Ware JE Jr, Sherbourne CD. The MOS 36-item short-form health survey (SF-36). I. Conceptual framework and item selection. Med Care. 1992;30(6):473-83.

87. Wichers M, Peeters F, Geschwind N, Jacobs N, Simons CJ, Derom C,... van Os J. Unveiling patterns of affective responses in daily life may improve outcome prediction in depression: a momentary assessment study. J Affect Disord. 2010;124(1-2):191-5. doi:10.1016/j.jad.2009.11.010.

88. Wilhelm P, Schoebi D. Assessing mood in daily life. European Journal of Psychological Assessment. 2007;23(4):258-67.

89. Wolfe F. Fibromyalgia. Rheum Dis Clin North Am. 1990;16(3):681-98.

90. Wolfe F, Clauw DJ, Fitzcharles MA, Goldenberg DL, Hauser W, Katz RS,... Winfield JB. Fibromyalgia criteria and severity scales for clinical and epidemiological studies: a modification of the ACR Preliminary Diagnostic Criteria for Fibromyalgia. J Rheumatol. 2011;38(6):1113-22. doi:10.3899/jrheum.100594.

91. Wolfe F, Clauw DJ, Fitzcharles MA, Goldenberg DL, Katz RS, Mease P,.. . Yunus MB. The American College of Rheumatology preliminary diagnostic criteria for fibromyalgia and measurement of symptom severity. Arthritis Care Res (Hoboken). 2010;62(5):600-10. doi:10.1002/acr.20140.

92. Wood PB. Role of central dopamine in pain and analgesia. Expert Rev Neurother. 2008;8(5):781-97. doi:10.1586/14737175.8.5.781.

93. Wood PB, Patterson JC 2nd, Sunderland JJ, Tainter KH, Glabus MF, Lilien DL. Reduced presynaptic dopamine activity in fibromyalgia syndrome demonstrated with positron emission tomography: a pilot study. J Pain. 2007;8(1):51-8. doi:10.1016/j.jpain.2006.05.014.

94. Wood PB, Schweinhardt P, Jaeger E, Dagher A, Hakyemez H, Rabiner EA,.. . Chizh BA. Fibromyalgia patients show an abnormal dopamine response to pain. Eur J Neurosci. 2007;25(12):3576-82. doi:10.1111/j.1460-9568.2007.05623.x.

95. Zautra AJ, Fasman R, Reich JW, Harakas P, Johnson LM, Olmsted ME, Davis MC. Fibromyalgia: evidence for deficits in positive affect regulation. Psychosom Med. 2005;67(1):147-55. doi:10.1097/01.psy.0000146328.52009.23.

96. Zautra AJ, Johnson LM, Davis MC. Positive affect as a source of resilience for women in chronic pain. J Consult Clin Psychol. 2005;73(2):212-20. doi:10.1037/0022-006X.73.2.212.

\section{Tables}

Table 1 Questionnaires at assessment (T0), at baseline (T1) and three months follow up (T2) 
Variables

\section{Sociodemographic and medical variables:}

Demographics: for example age, marital, status, education $\quad x$

Medical history: for example duration of FM, medication, treatment $\quad x$

Lifestyle and health behaviour:

smoking history, alcohol consumption, exercise

\section{Psychometric data:}

M.I.N.I International Neuropsychiatric Interview

Beck Depression Inventory (BDI, Beck, 1961)

State-Trait Anxiety Inventory (STAI, Laux 1981)

Profile of Mood States, POMS

Quality of Life, WHOQOL, (Skevington et al. 2004)

Fibromyalgia questionnaire revised (FIQ-R), (Bennet, 2009)

Sleep quality, medical outcomes study sleep scale MOS (Stewart, 1988)

Five Facet Mindfulness Questionnaire (Baer, 2006)

Pain Coping Questionnaire (Brown \& Nicassio, 1987)

Cognitive Emotion Regulation questionnaire (CERQ), (Garnefski, 2007)

Savouring beliefs inventory, (Bryant, 2003)

Temporal Experience of pleasure scale (Gard, 2006)

Hamilton Rating Scale for Depression

Edinburgh handedness Inventory

\section{Pain related outcomes:}

Brief Pain Inventory (BPI, Cleeland, 1994)

Verbal Rating Scale for current Pain Intensity from SF-36 (Ware, 1992)

Pain Disability Index (Dillmann, 1994)
T0

T1

T2

T3

$x$

$\mathrm{X}$

$\mathrm{x}$

$x$

$\begin{array}{lll}x & x & x \\ x & x & \\ x & x & x \\ x & x & x \\ x & x & x \\ x & x & x \\ x & x & x \\ x & x & x \\ x & x & x \\ x & x & x \\ x & x & x\end{array}$

$x$

$\mathrm{x}$

Table 2: schedule of enrollment, interventions, and assessments

From: Understanding and restoring dopaminergic function in Fibromyalgia patients using a mindfulnessbased psychological intervention: A [18F]-DOPA PET study: Study protocol for the FIBRODOPA study-a randomized controlled trial 


\begin{tabular}{|c|c|c|c|c|c|}
\hline & $\begin{array}{c}\text { From } \\
\text { September } \\
2020\end{array}$ & $>1$ day & $>2$ days & $\begin{array}{c}\text { After } 8 \text { weeks } \\
\text { MORE } \\
\text { intervention/ } \\
\text { waitlist } \\
\end{array}$ & \\
\hline TIMEPOINT** & $-t_{1}$ & 0 & $t_{1}$ & $T_{2}$ & $T_{3}$ \\
\hline ENROLMENT: & $\begin{array}{l}\text { Information } \\
\text { Screening }\end{array}$ & & $\begin{array}{c}\text { Pre-test } \\
\text { measures }\end{array}$ & $\begin{array}{c}\text { Post-test } \\
\text { measurements }\end{array}$ & $\begin{array}{l}\text { 3-Months } \\
\text { follow up }\end{array}$ \\
\hline Eligibility screen & $\mathrm{x}$ & & & & \\
\hline \multirow{2}{*}{$\begin{array}{r}\text { Informed consent } \\
\text { Baseline data }\end{array}$} & $\mathrm{x}$ & & & & \\
\hline & $x$ & & & & \\
\hline Allocation & & $x$ & & & \\
\hline \multicolumn{6}{|l|}{ INTERVENTIONS: } \\
\hline F-DOPA PET scan & & & $\mathbf{x}$ & $X$ & \\
\hline MR scan] & & & $\mathrm{x}$ & $\bar{x}$ & \\
\hline Reward task & & & $\mathrm{x}$ & $\bar{x}$ & \\
\hline \multirow[b]{2}{*}{$\begin{array}{r}\text { ASSESSMENTS: } \\
\text { Medical history } \\
\text { Clinical psychiatric } \\
\text { interview } \\
\text { Physical } \\
\text { examination, } \\
\text { tender point } \\
\text { evaluation } \\
\text { Self-report scales }\end{array}$} & & & & & \\
\hline & $x$ & & & & \\
\hline \multirow{2}{*}{$\begin{array}{r}\text { Intervention } \\
\text { related clinical } \\
\text { outcomes }\end{array}$} & & & $\mathrm{x}$ & $\mathrm{x}$ & $\mathrm{x}$ \\
\hline & & & $x$ & $\mathrm{X}$ & \\
\hline
\end{tabular}

Figures 

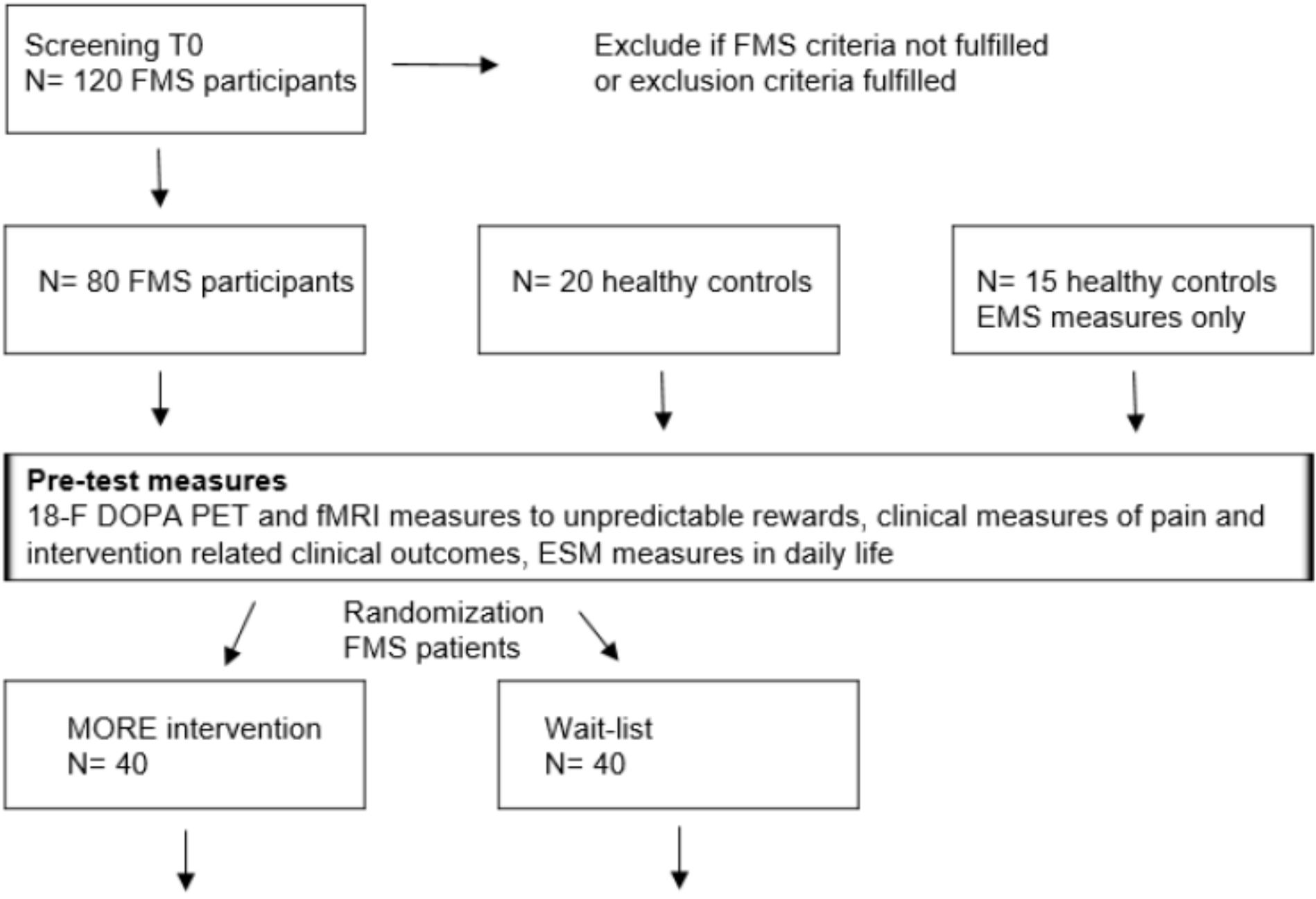

\section{Post-test measures}

18-F DOPA PET and fMRI measures to unpredictable rewards, clinical measures of pain and intervention related clinical outcomes, ESM measures in daily life

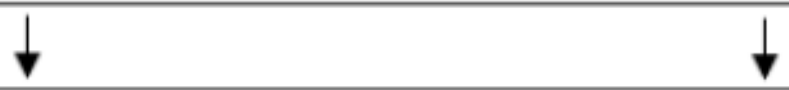

3-months follow-up

Clinical measures of pain and intervention related outcomes

Figure 1

Flowchart of the intervention study

\section{Supplementary Files}

This is a list of supplementary files associated with this preprint. Click to download.

- SPIRITchecklistfinal.docx 